\title{
Internet of Things for the Hotel Industry: A Review
}

\author{
Sérgio Tenreiro de Magalhães ${ }^{1,2,3}$, M. J. Magalhães ${ }^{4,5}$, and Kenneth Revett ${ }^{6}$ \\ ${ }^{1}$ ALGORITMI Reseach Center - University of Minho, Guimarães, Portugal, \\ psmagalhaes@dsi.uminho.pt \\ ${ }^{2}$ Faculdade de Filosofia e Ciências Sociais da Universidade Católica Portuguesa, Braga, \\ Portugal, stmagalhaes@braga.ucp.pt \\ ${ }^{3}$ Department of Computer Science, University of Beira Interior, Covilhã, Portugal, \\ stmagalhaes@di.ubi.pt \\ ${ }^{4}$ GOVCOPP, University of Aveiro, Aveiro, Portugal, mjmagalhaes@ua.pt \\ ${ }^{5}$ Faculdade de Filosofia e Ciências Sociais da Universidade Católica Portuguesa, Braga, \\ Portugal, mjmagalhaes@braga.ucp.pt \\ ${ }^{6}$ Anastamose International, Boston, MA USA, Ken.revett@gmail.com
}

\begin{abstract}
The Internet of Things (IoT) represents an opportunity for the hotel industry to increase customer satisfaction while simultaneously reducing operational costs. This paper analyses the existing knowledge on this subject, through a review of the relevant publications indexed by Scopus and/or ISI Web of Science, concluding that, despite the existence of many relevant patents, registered in the past few years, the published research is very limited on this topic. The restriction to publication prior to the conclusion of the patent registration process may be a justification for this fact, and, if so, the near future will bring many novelties that will help the development of the hotel industry. It is also possible to conclude from this work that the potential of IoT is not yet well explored in the hotel industry, once authors frequently theorize on the use of IoT for applications that could easily be of interest for the hotel industry, but fail to identify that opportunity as a major market.
\end{abstract}

Keywords: Internet-of-Things, Hotel industry, Hospitality, Pervasive Computing. 


\section{Introduction}

Managing revenues in the hotel industry is complex and dependent on the available information systems and on the characteristics of the facilities that exist on the hotels, once it consists of three components: pricing, revenue management and product distribution [1]. Pricing includes segmentation of costumers based on their needs, characteristics and willingness to pay; Revenue management, is the optimization of the mix of clients to maximize the revenues, which takes in consideration aspects like rate, length of stay and arrival date; Finally, product distribution relates to selling through the most adequate channels (company's website, Global Distribution Systems like Amadeus, Galileo and Sabre, online travel sites, etc.), which is related to the description of the available profits [1]. Therefore, the available possibilities for the definition of the clients mix relates to the type of products that are available, which includes, for instance, mini-bar, wi-fi access and cable TV. These, among others, participate in the operational costs of hotels, but also participate in pricing. Varini et al. found that "computer equipment" is a characteristic much valued by hotel clients travelling on business, similar to the importance of breakfast (lower than price, but much higher than bathroom amenities, TV, fitness equipment, fruits and water or coffee and tea making facility in the room) [2].

Information and Communication Technologies (ICT) have an important role in hotel management and in hotel success as a business. Sirirak et al found that ICT availability and integration has a significant positive influence in operational productivity and that the intensity of ICT usage has a significant positive impact in both operational productivity and customer satisfaction [3].

Another relevant operational cost in hotels in related to theft. Many clients steal small items from the hotel, like ashtrays, glasses or towels, and sometimes employees steal more significant items, once they are familiarized with security procedures and have access to restricted zones and exits [4].

Despite some privacy concerns associated with Internet-of-Things (IoT) technologies [5] [6] [7], IoT can have a relevant role in hotel management, both in increasing the customer's experience as in decreasing operational costs and theft related costs. This paper reviews the existing literature on this subject, focusing both on contents and on the research itself, and the patents related bot to Internet of Things and the hotel industry, focusing on the number of papers and on the evolution on the research on this field of study.

The next section presents the method used to collect relevant works and patents and is followed by the conclusions found, both on the level of research interest in this topic as in the contents of the published works. Finally, in the last section, the conclusions are presented. 


\section{Method}

For this work we made several queries in Scopus and Web-of-Science, the two most relevant indexers in peer-reviewed academic publishing in the beginning of September 2016.

In the Scopus search portal, the queries were made using the terms presented in Table 1, without excluding any subject areas but excluding one work that was a conference review. The authors also intended to exclude any books, reviews or editorials, due to the nature of these type of scientific productions, but the results did not include any works of those types. The key term "Internet of Everything" was included following the adoption by CISCO of this term, but no results were found using this term.

An interesting fact is that the searches using the key terms "IOT" and "Internet of Things" combined with the key term "hotel" resulted in different result sets (both with 10 works) and the searches using the key terms "IOT" and "Internet of Things" combined with the key term "hospitality" also resulted in different result sets. The term "hospitality" is much includes much more than hotels, but the choice was made to include it in the study once there was no other efficient way to capture studies that were destined to "hotel like" facilities (e.g. hostels or any other lodging facilities).

Combining all the searches, through an OR operator, resulted in a total of 14 papers.

The same queries were made to look for patents. The number of patents found is presented in Table 2 and the combined search resulted in 353 distinct patents

Table 1. Search queries and number of papers found in Scopus

\begin{tabular}{lc}
\hline \multicolumn{1}{c}{ Terms used in the query } & Number of documents found \\
\hline "Internet of Things" \& Hotel & 10 \\
"Internet of Things" \& Hospitality & 2 \\
IOT \& Hotel & 10 \\
IOT \& Hospitality & 3 \\
"Internet of Everything" \& Hotel & 0 \\
"Internet of Everything" \& Hospitality & 0 \\
\hline
\end{tabular}

Table 2. Search queries and number of patents found in Scopus

\begin{tabular}{lc}
\hline Terms used in the query & Number of patents found \\
& \\
\hline "Internet of Things" \& Hotel & 302 \\
"Internet of Things" \& Hospitality & 52 \\
\hline
\end{tabular}




\begin{tabular}{lc}
\hline IOT \& Hotel & 150 \\
IOT \& Hospitality & 8 \\
"Internet of Everything" \& Hotel & 0 \\
"Internet of Everything" \& Hospitality & 0 \\
\hline
\end{tabular}

The next stage was to read the abstracts in order to eliminate papers that, despite the use of the relevant keywords in their title, abstract or paper keywords, were clearly out of this work's scope. This lead to the exclusion of two paper that, despite being classified as journal papers, were a "Conference Preview" and a conference announcement, leaving 12 papers for a deeper analysis.

The procedure was replicated in the Web-of-Science search portal, making the queries in the "Topic" fields (title, abstract and keywords) without excluding any scientific domains. The number of papers found is presented in Table 3 and the number of papers found that were not indexed by Scopus is presented in Table 4.

Table 3. Search queries and number of papers found in Web of Science

\begin{tabular}{lc}
\hline \multicolumn{1}{c}{ Terms used in the query } & Number of papers found \\
\hline "Internet of Things" \& Hotel & 3 \\
"Internet of Things" \& Hospitality & 1 \\
IOT \& Hotel & 4 \\
IOT \& Hospitality & 1 \\
"Internet of Everything" \& Hotel & 0 \\
"Internet of Everything" \& Hospitality & 0 \\
\hline
\end{tabular}

Table 4. Search queries and number of papers found in Web of Science and not in Scopus

\begin{tabular}{ll}
\hline \multicolumn{1}{c}{ Terms used in the query } & Number of papers found \\
\hline "Internet of Things" \& Hotel & 0 \\
"Internet of Things" \& Hospitality & 1 \\
IOT \& Hotel & 0 \\
IOT \& Hospitality & 0 \\
"Internet of Everything" \& Hotel & 0 \\
"Internet of Everything" \& Hospitality & 0 \\
\hline
\end{tabular}

The next stage was to read the abstracts in order to eliminate papers that, despite the use of the relevant keywords in their title, abstract or paper keywords, were clearly out of this work's scope. This lead to the exclusion of two papers. 
In the first paper excluded [8], the authors analyze the potential of IOT in tourism various applications such as monitoring and location of animals, flora monitoring, creating itineraries mediated by IOT (with connected devices that communicate with the tourist when he is in the vicinity), marketing and hospitality services. None of these applications has direct applicability in the hotel industry.

The second paper excluded [9] presents a solution for the monitoring of food quality during transport (eventually having hospitality businesses as the final destination).

All together 11 papers were analyzed and reviewed.

On the 11 relevant papers found, two studies were made: the first on the scientific domain, through a study of the types of publication, countries of the authors and evolution through time of the number of publications; the second on the contributions of these papers, in order to summarize the respective state-of-art. This work also presents the evolution of the number of patents registered. The obtained results are presented in the next section.

\section{Results}

\subsection{The evolution of patents registered}

The number of registered patents is a good indicator of the importance to the industry of the scientific developments. On the relevant topic, there has been a growing interest in this domain, expressed by a large number of patents (353, according to Scopus) registered, all in the past 4 years. Figure 1 shows a clear increase in the number of patents, evolving from 11 in 2012 to 163 in 2016 (from January to mid-September only). There is, therefore, little doubt that the Internet of Things is perceived as much relevant to the hotel industry and has commercial perceived value (enough to justify the protection of the inventors' rights through a patent, which is costly).

Fig. 1. Evolution of the number of patents registered 


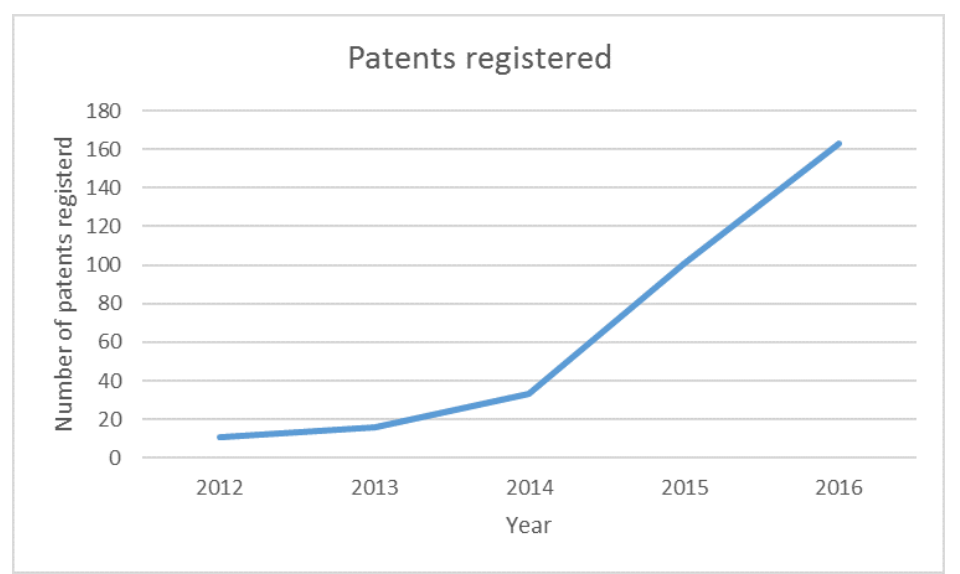

\subsection{The evolution of published research on the topic}

All the papers found were published since 2013, showing a recent and increasing interest on the topic, which can be seen through the tendency of evolution along the years in the number of publications (Figure 2).

Fig. 2. Evolution of the number of papers published

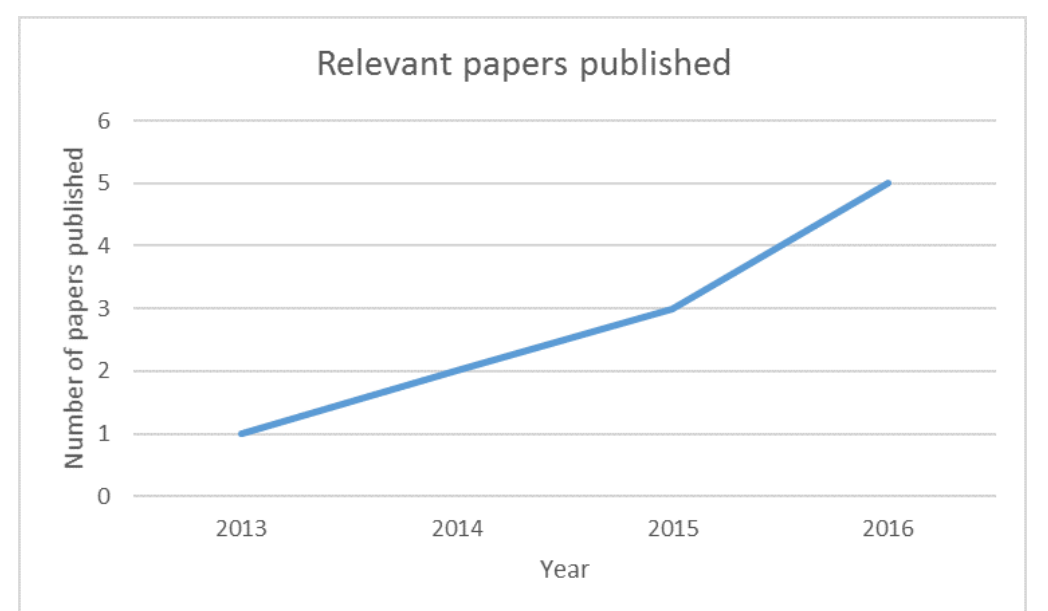

The topic has captured the interest of researchers worldwide, with researchers from eight countries and three continents (America, Asia and Europe) contributing 
to the development of the knowledge in this field (Figure 3$)^{1}$. It is also symptomatic that the contributions come from six different scientific domains (Figure 4) ${ }^{2}$.

The limitations to academic publishing prior and during a patent registration process might explain, at least partly, the relatively small number of published works on this topic, especially when compared to the number of registered patents. If this hypothesis turns out to be correct, the next few years will result in a boom of published papers that will transform the hotel industry, which has to meet the demanding requirements of the XXI century.

Fig. 3. Papers published by country of origin

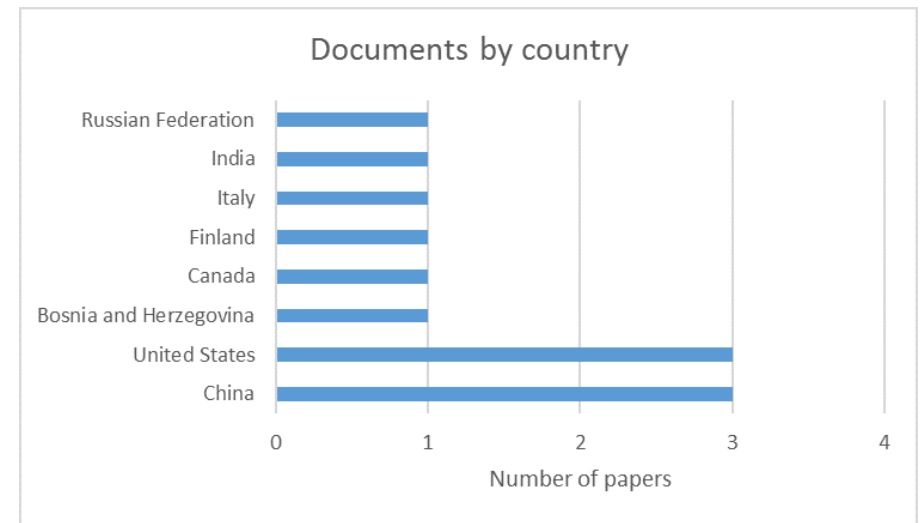

Fig. 4. Subject areas of classification of the papers analysed.

\footnotetext{
${ }^{1}$ Note that some papers have authors from more than one country and some countries are repeated in many papers. Therefore, the sum of number of papers per country is different than the total number of papers analyzed.

${ }^{2}$ Note that some papers are classified in more than one subject area. Therefore, the sum of number of papers in the subject areas is bigger than the total number of papers analyzed.
} 


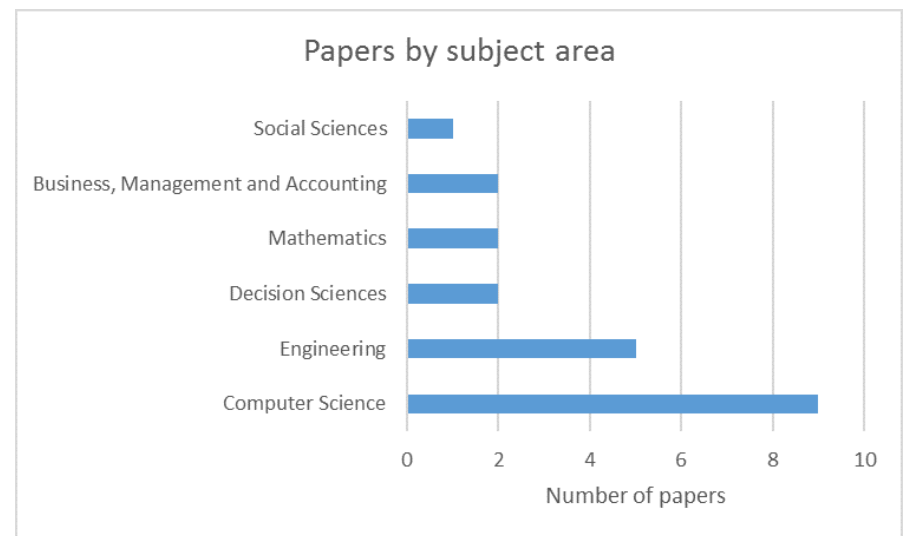

\subsection{Contributions found}

The contributions of the 11 papers study fall into five categories: marketing, technical implementations, business transformation, proposal of innovative products and risk management, as presented in the next subsections.

\section{Marketing}

Two papers addressed the importance of the IoT to the marketing strategies in the hotel industry.

In [10], the authors, focused on smart tourism destinations, studied the ecosystems of the Italian destinations of Elba, Gallura and Livigno, which have similar tourism industries, in number of tourism business and in number of visits (around 500.000 per year). Each one of those ecosystems was considered to be a net with two subnets: one constituted by the real tourism businesses; and the other constituted by their virtual representation in the form of a website. The businesses constituted the nodes and their connections represent business agreements, coownership, partnerships or common participation in associations or consortiums. The study of the corresponding graph allowed the authors to conclude that the combination of real-life and virtual elements was the best strategy for the dissemination of ideas, concluding that hotels should focus their marketing strategies in a way that combines the real life with the virtual world. The authors consider IoT to be one of the pillars of this new virtual strategy but did not elaborate much on that aspect.

In [11] the authors address the issue of over-information of users, as a result of an interconnected world through the IoT and propose a recommendation system. The proposed system is a kind of decision support system for the ordinary citizen, 
including the recommendation of hotels. This is an important article for a perspective of what may be the hotel marketing in a world of IOT.

In [12], Balandina and colleagues present and discuss some innovative solutions for e-Tourism developed on top of the Open Source platform for location bases services called Geo2Tag. The paper is not particularly focused on the hotel industry, focusing mostly in museum related situations, but do propose an extension to the TripAdvisor ecosystem that could be more efficient than a printed stick in the entrance hall. The main idea was to use an IoT-Tag that would broadcast the Trip-Advisor rating of the hotel and any other information that could help to attract more consumers, like the menu or any special offers. This paper also theorizes on some other relevant applications for e-Tourism, some of which, despite not being presented in that way in the paper, could be interesting for the hotel industry (e.g. monitoring the quality of the air or the air temperature).

\section{Technical Implementation}

The paper presented by Chang-Le Zhong et al [13] focus on technical aspects of IoT implementations. The authors present a five layers' system architecture for IoT and a technical solution for the design of an IoT application scheme using the IoT gateway as a bridge, which was thought to meet the demands of the hotel industry to improve the guest's living environment.

\section{Proposal of Innovative Products}

In [14] the authors present an IoT based solution for hotels to provide meals adequate to their guests' nutritional needs. The solution is also adequate for home use, but is best suited for the hotel industry, due to the flexibility associated with the co-existence of alternative meals and ingredients, that create a set of possible choices that are not typically available in home meals. With the proposed solution it is possible for the client to define the meal based on he's own nutritional needs, both in type and in quantity. This is also relevant for guests with diet restrictions.

\section{Risk Management}

As mentioned in the introduction, there are some security/safety/privacy concerns related to the IoT. Therefore, it is not surprisingly that three papers address this issue.

Considering that the IoT increases the risk of a cybernetic attack to have direct impact on the physical world, the study presented in [15] introduces a security framework, named SecIoT, for IoT infrastructures, which is based on the security requirements of three different environments, including hotels. In their studies, the authors concluded that IoT security is a serious concern of the users. 
Considering the risks associated with the increasing number of computational devices that are interconnected, the authors of [16] propose a model for their integration designed to assure that the automatic decisions that occur based on the information flow are the most adequate. In the same line of thought $\mathrm{Yi} \mathrm{Xu} \mathrm{[17]} \mathrm{con-}$ siders that without an ecosystem and a scalable architecture it will be extremely difficult to manage a network of IoT enabled devices, which represents, at least, an operational hazard. Therefore, he presents a proposal for an architecture designed to meet the scalability requirement of an IoT network.

\section{Business Transformation}

The work of Ning Wang [18] discusses the construction of a Smart Tourism perception system and management platform, based on the assumption, sustained by the author, that the development of Smart Tourism, or of a smarter tourism, based on the IoT, aims to provide to all types of tourists a more convenient intelligent travel experience and to provide the tourism business with more efficient and intelligent information platforms while, simultaneously, allowing a deeper development of the resources.

Another interesting paper, regarding the potential of IoT to transform the hotel industry, is [19]. This study presents several IT applications to the hotel industry, considering that IOT has potential of use for "real-time customer service, customer information identification, service guidance, route planning and customer expense recording", among other non-specified applications.

In [20], the authors discuss the need for hotels to include in their business model the transformation of reality resulting from the advent of ubiquitous computing/IOT, concluding that there is an opportunity for increased income by reengineering their operating models and their physical and logic connectivity models, through the creation of services to their usual customers, for local residents and for transient visitors. The authors argue that hotel industry could increase its sustainability by taking advantage of its potential to accommodate new sources of income, such as demonstration centers designed to present for passers products and/or services sensitive to the location; and by using IoT to increase the efficiency of their marketing strategy. The authors also argue that an integrated communication system based on IOT can actively improve customer satisfaction with comfort solutions customized to the clients (e.g. intensity/color of the room lighting, room temperature or even bed linen, through the use of smart blankets). The paper proposes an architecture that also enables a more efficient management of resources by the hotels. The authors further state that, in addition to the management challenges in a competitive environment, coupled with the refusal of integration of IOT, "hotels risk alienating Millennials, the growth segment of the market, by failing to adopt the model that seamlessly integrates all of the potential benefits of pervasive connectivity with a fully articulated sustainable travel ethos". 


\section{Conclusions}

Despite the growing interest on the subject of this work, demonstrated by the registration of patents that relate IoT and the hotel industry, there aren't many relevant indexed published works. These facts can even be interrelated, once there are restrictions to what can be published about a novelty before registering a patent. Nevertheless, the literature review found contributions in five categories: marketing, technical implementations, business transformation, proposal of innovative products and risk management, which can be very important for the development of the hotel industry in the next few years, especially if the hypothesis that the reduced number of publications is related to the registration of patents gets confirmed. If so the next years will produce a substantial increase in the published knowledge on this subject.

It is also possible to conclude that the potential of IoT is not yet well explored in the hotel industry, once authors frequently theorize on the use of IoT for applications that could easily be of interest for the hotel industry, but fail to identify that opportunity as a major market.

Acknowledgments This work has been supported by COMPETE: POCI-01-0145-FEDER007043 and FCT - Fundação para a Ciência e Tecnologia within the Project Scope: $\mathrm{UID} / \mathrm{CEC} / 00319 / 2013$

\section{References}

[1] B. Vinod, "Unlocking the value of revenue management in the hotel industry," Journal of Revenue and Pricing Management, pp. 178-190, 2004.

[2] K. Varini, R. Engelmann, B. Calessen and M. Schleusener, "Evaluation of the pricevalue perception of customers in Swiss hotels," Journal of Revenue and Pricing Management, vol. 2, pp. 47-60, 2003.

[3] S. Sirirak, N. ISlam and D. B. Khang, "Does ICT adoption enhance hotel performance?," Journal of Hospitality and Tourism Technology, vol. 2, pp. 34-49, 2011.

[4] S. Irini and R. Prus, "Doing Security Work: Keeping Order in the Hotel Setting," Canadian Journal of Criminology, vol. 24, pp. 61-82, 1982.

[5] C. Sousa, P. Pereira, S. Tenreiro de Magalhães, L. Santos and H. Santos, "RFID: Big Brother Global?," in Proceedings of the 8th European Conference on Information Warfare and Security, Lisbon, 2009.

[6] E. Kelly and G. S. Erickson, "RFID tags: commercial applications v. privacy rights," Industrial Management \& Data Systems, vol. 105, pp. 703-713, 2005.

[7] B. Turner, "When Big Data Meets Big Brother: Why Courts Should Apply United States v. Jones to Protect People's Data," North Carolina Journal of Law \& Technology, vol. 16, pp. 377-527, 2015.

[8] O. Gcaba and N. Dlodo, "The internet of things for South African tourism," Durban, 2016.

[9] Maksimović, Mirjana, V. Vujović and E. Omanović-Mikličanin, "A low cost internet of things solution for traceability and monitoring food safety during transportation," in 7th In- 
ternational Conference on Information and Communication Technologies in Agriculture, Food and Environment, Kavala; Greece, CEUR-WS, 2015.

[10] G. Del Chiappa and R. Baggio, "Knowledge transfer in smart tourism destinations: analyzing the effects of a network structure," Journal of Destination Marketing \& Management, p. (in press), 2015

[11] Lee, Sunhwan, Chandra, Anca and Jadav, Divyesh, "An empirical study on hybrid recommender system with implicit feedback," in 20th Pacific-Asia Conference on Advances in Knowledge Discovery and Data Mining, Auckland; New Zealand, Springer, 2016.

[12] Balandina, Ekaterina, S. I. Balandin, Koucheryavy, Yevgeni A. and Mouromtsev, Dmitry I., "Innovative e-Tourism Services on Top of Geo2Tag LBS Platform," in Proceedings - 11th International Conference on Signal-Image Technology and Internet-Based Systems, SITIS, Bangkok; Thailand, IEEE, 2015.

[13] C.-L. Zhong, Z. Zhu and R.-G. Huang, "Study on the IOT architecture and gateway technology," in 14th International Symposium on Distributed Computing and Applications for Business Engineering and Science, IEEE, 2015.

[14] W. Minghua, Y. Liuqing, Z. Qiaoling and Z. Jingui, "Construction of optimal allocation system on the edible agricultural products' components of the nutrition and health care based on internet of things," in 2013 International Conference on Precision Mechanical Instruments and Measurement Technology, Shenyang, Liaoning; China, 2013.

[15] X. Huang, H. Lin and Z. Yan, "SecIoT: A security framework for the Internet of Things," Security and Communication Networks, p. (in press), 2015.

[16] P. Raj, K. V. L. S. Sampath and V. Skylab, "Open Data and Messaging Standards: Enabling On-The-Go Operation of IT and Non-IT Resources via IoT Devices," Bangalore, 2015.

[17] Y. Xu, "Scalable Cloud-Sensor Architecture for the Internet of Things," IEEE Internet of Things Journal, vol. 3, pp. 285-298, June 2016.

[18] N. Wang, "Research on construction of smart tourism perception system and management platform," in 2014 International Conference on Manufacturing Technology and Electronics Applications, Taiyuan; China, Trans Tech Publications Ltd, 2014, pp. 1745-1748.

[19] L. Yunpeng, X. Yongqiu and H. Chao, "Promoting hotel managerial and operational level with new technology," in 2013 International Conference on Vehicle and Mechanical Engineering and Information Technology, Zhengzhou, Henan; China, 2013.

[20] Hecht, Howard, Mayier, Mahemuti and Perakslis, Christine, "Pervasive connectivity: The thriving hotel of the future," in 2014 IEEE International Conference on Pervasive Computing and Communication Workshops, Budapest; Hungary, IEEE, 2014. 